Niño, L.E. \& Gama, A. (2013). Los estándares en el currículo y la evaluación: ¿relaciones de medición, control y homogenización o, posibilidad de formación, diversidad y evaluación crítica?. Revista Electrónica Interuniversitaria de Formación del Profesorado, 16 (3), 163-176.

DOI: http://dx.doi.org/10.6018/reifop.16.3.186781

\title{
Los estándares en el currículo y la evaluación: ¿relaciones de medición, control y homogenización o, posibilidad de formación, diversidad y evaluación crítica?
}

\author{
Libia Stella Niño Zafra, Antonio Gama Bermúdez \\ Universidad Pedagógica Nacional de Colombia
}

\section{Resumen}

Este artículo presenta la investigación homónima cuyo objetivo fue construir conocimiento social, político y pedagógico sobre los estándares, el currículo y la evaluación, así como analizar las relaciones y posibilidades de control, homogenización, formación o diversidad que estas relaciones promueven en las instituciones educativas. El estudio comprendió el análisis de las concepciones dadas por los profesores situados y expertos nacionales e internacionales a la categoría estándar, con el fin de caracterizar las implicaciones y consecuencias que su normatividad en la práctica pedagógica de dos colegios distritales, generando escenarios de prácticas alternativas en las instituciones escolares

\section{Palabras clave}

Estándares; Evaluación; Investigación Cualitativa; Conocimiento social y político.

\section{Contacto:}

Libia Stella Niño Zafra, libistel@gmail.com, Universidad Pedagógica Nacional de Colombia.

Este artículo corresponde a la investigación realizada por el Grupo Evaluándo_nos: Pedagogía crítica, Docencia y Evaluación, conformado por Libia Stella Niño (Coordinadora), Alfonso Tamayo Valencia, Nubia Conde Quintero, José Emilio Díaz, Antonio Gama Bermúdez, y las monitoras de investigación Claudia Luna y Johana Jiménez. Al grupo investigador se sumó la profesora Gladys Soler de la Universidad de Cundinamarca para el desarrollo de esta investigación... La puesta en acción se amplió con la participación en los talleres de 140 maestros de los dos colegios distritales mencionados. 


\title{
Standards in curriculum and assessment: measuring relationships, control and homogenization or training opportunity, diversity and critical assessment?
}

\begin{abstract}
The project was approached with the interest of building social political and pedagogical knowledge, about standards, curriculum and assessment on education, and to analyze the relationships of control, homogenization, diversity training that this relationships promote in the educational institutions. The study aimed to analyze and understand the concepts given by the teachers and national and international experts, to the standard and to characterize the implications and consequences of its policies have had on the pedagogical practice of District Schools. The analysis is based on the inclusion of the contexts in which they carried out the pedagogical work and the recognition of subjects in ethical-political dimension, for the generation of alternative practices in the schools.
\end{abstract}

\section{Key words}

Standards, Assessment, Qualitative Research, Social political and pedagogical knowledge.

\section{Introducción}

El presente artículo de investigación sintetiza el avance teórico-práctico en la comprensión y análisis del sentido asignado por los profesores a la normatividad sobre los Estándares Básicos de Competencias, en el estudio llevado a cabo en dos colegios públicos del Distrito Capital: las instituciones educativas República de China y Heladia Mejía, así como la revisión de las reflexiones calificadas de seis académicos nacionales e internacionales sobre el significado, las implicaciones y las consecuencias que tales normatividades conllevan sobre el currículo y la evaluación.

Para el Grupo de Investigación Evaluándo_nos el interés de este proyecto ha sido indagar la manera en que los profesores han asumido los estándares y comprender las acepciones dadas a la categoría estándar, sus relaciones con el currículo y la evaluación; además de caracterizar las implicaciones y consecuencias que las políticas de estandarización han tenido en la práctica pedagógica de los Colegios Distritales.

Al Equipo Investigador, contar con investigaciones sobre currículo y evaluación, en estos y otros colegios del Distrito Capital, le ha permitido constatar el predominio de un enfoque técnico-instrumental, que refuerza la visión de currículo como plan de estudios, la evaluación como práctica de medición y los estándares como referentes mínimos de planeación, demandando de la comunidad académica e investigativa la necesidad de estudiar desde otras perspectivas teóricas y prácticas, posibles alternativas en la manera de abordar el uso y significado de los mismos. Así, el proyecto asumió como práctica metodológica la perspectiva de indagación cualitativa propuesta por Eisner (1998), con el propósito de hacer una crítica educativa a las opiniones y planteamientos del Ministerio de Educación Nacional - MEN, los profesores de los colegios y los expertos nacionales e internacionales, quienes de forma escrita expresaron los puntos de vista que fueron socializados y discutidos mediante la estrategia pedagógica de la realización de talleres. 
Con este propósito, en el análisis se han seguido las etapas de la investigación evaluativa desde la descripción hasta la tematización, para consolidar la formulación de ideas, que se han constituido en hilos conductores de lo expresado en los talleres. Estos mecanismos teórico-prácticos permitieron el trabajo colectivo con los profesores de las instituciones participantes quienes, a la vez que brindaban sus aportes, construían nuevos significados a estas categorías de estudio: estándares, currículo y evaluación.

\section{Dimensión teórica: estándares en educación}

En el marco del discurso de la calidad de la educación se plantean pruebas masivas censales, estándares, test y modelos curriculares normatizados, debido a que calidad de la educación y estándar son categorías surgidas del mundo empresarial, convergentes desde la racionalidad técnica que prioriza resultados medibles y evidenciables, en coincidencia con lo expresado por Santos Guerra (2003). En Estados Unidos, se inicia en los negocios y empresas para uniformar patrones de medida, por lo que el término estándar es comprendido como un criterio que permite asumir un nivel de calidad en un producto o situación que se espera generalizar, usualmente puestos en el mercado. Ambos términos son yuxtapuestos a la educación desde los discursos de la industria y la economía que apoyan procesos de mejora al alcanzar consensos sobre lo que se espera de la producción.

A juicio de Ravitch (1995), la creación de los estándares nacionales en Estados Unidos en 1990 recibe críticas debido a las siguientes características: 1. Los estándares nacionales 'deben ser mínimos, reducidos a su más bajo común denominador, especialmente si ellos son controlados por una agencia federal'. Este control es real, especialmente cuando son elaboradas por un Consejo Político, más aún cuando las Leyes tienen una representación histórica en pobres desempeños en la educación. Esta crítica puede ser cierta en cuanto que una reducción de los estándares a mínimos desempeños de estudiantes y docentes sobre su hacer, pudiera llegar a esquematizar los procesos pedagógicos que se dan en las instituciones. 2. El gobierno puede llegar a imponer valores y opiniones controversiales. Los críticos se refieren a que si el Gobierno gana control sobre el currículo y los test, esto puede conllevar que las personas se adapten y se conformen con esas ideas políticas e ideológicas, controlando las mentes de los estudiantes. 3. Los estándares nacionales basados en temas tradicionales y disciplinas como matemáticas, ciencias e historia van a limitar el currículo. Los críticos afirman que los estándares nacionales hacen rígido el currículo, por lo cual no tratan problemas de la vida real o de una manera interdisciplinar y solo unidisciplinar, en lugar de abordar temas que partan del contexto por materias o cursos. 4. Los test nacionales van a afectar a los niños y distorsionar las prioridades del aula de clase. La crítica siente que imponiendo los estándares se va a trivializar la enseñanza, uniformando los salones de clase en todo el país, reduciendo el aprendizaje a la preparación para los test. 5. Los estándares nacionales no van a hacer nada para ayudar a las escuelas públicas de la ciudad, pues se argumenta que la más urgente necesidad de las escuelas públicas es dinero y no el seguimiento de estándares. Apple denunció los estándares como una reforma barata que acrecentará las diferencias de raza, género y nivel económico al no atender a estas problemáticas (Ravitch, 1995). 6. Los estándares nacionales y evaluación de desempeños no van a expandir la igualdad de oportunidades. Los críticos se quejan de que los estándares nacionales y la evaluación no mejorarán el desempeño de las minorías ni va reducirán la brecha entre estudiantes de minorías y no minorías, ya que los altos estándares evitan este proyecto de equidad. 7. Los estándares nacionales y de la evaluación no van a mejorar el desempeño porque la mayoría de los profesores los ignoran y va a hacer lo que ellos siempre han hecho. 8. El fracaso de los estándares nacionales y test de desempeño van a poner en 
detrimento la fe sobre la educación pública, lo que favorecerá la vía de la privatización para la educación. La crítica culpa a los organismos reguladores de las políticas de limitar la información acerca del desempeño, por lo que también se afectarían las percepciones de los padres sobre la educación, lo que acrecentaría la demanda sobre la educación privatizada. 9. Los estándares nacionales y la evaluación de desempeño realizarán muy poco por ellos mismos.

Estas críticas a la proclamación de estándares curriculares en educación, quince años después, son aceptadas por la misma Ravitch debido a que el establecimiento de mínimos con los estándares nacionales ha llevado a que se enfatice en las actividades de lectura y cálculo, como sucede en las escuelas privadas de Estados Unidos, Escuelas Charter. En dicho contexto, Bigelow (2010), HueyShanTan (2010) y Cohen (2010) muestran que los estándares hacen al currículo rígido, al limitar el abordaje de problemas de la vida real, acrecentando las diferencias de raza, clase y género, como lo expresa Apple (1995), en detrimento de la fe en la educación pública, debido a que las escuelas privadas refuerzan el desempeño en los resultados en las pruebas federales sin permitir el conocimiento sobre los resultados académicos que se pueden comparar con este sector educativo.

En su estudio sobre los estándares, el Grupo Evaluándo_nos (2006) destaca que este término ha transitado en la educación en busca de la calidad educativa siendo la idea más generalizada que hace parte de las políticas educativas en los países latinoamericanos, y se centra en que el nivel de calidad educativa se logra determinar a partir de los resultados de los estudiantes en pruebas estandarizadas nacionales (ICFES, ECAES, SABER) o internacionales (TIMSS -Estudio Internacional de las Matemáticas y de las Ciencia, PIRLS Estudio sobre el Progreso Internacional de Competencia en Lectura). Según Vasco (2003), en Estados Unidos, los estándares en matemáticas son formulados a mediados de los años 80 s por parte del National Council of Teachers of Mathematics - NCTM (Consejo Nacional del Profesores de Matemáticas), con el propósito de juzgar la calidad del currículo de matemáticas o un método de evaluación. Asimismo, "el Ministerio de Educación Nacional de Colombia, bajo el argumento de mejorar la calidad, y con los propósitos de 'proporcionar a todos los estudiantes las mismas oportunidades de aprendizaje' y 'contar con un referente común que asegure a todos el dominio de competencias básicas y los conceptos para vivir en sociedad y participar en ella en igualdad de condiciones"' (Vasco, 2003: 5), determina para la Educación Preescolar, Básica y Media, los Estándares curriculares para las áreas de matemáticas, lengua castellana, ciencias naturales y educación ambiental. Desde esta perspectiva, los estándares deben estar siempre sujetos a verificación y contribuir a la construcción de sistemas y procesos de evaluación interna.

Más que pensar en seguir uniformando según estándares, podría pensarse en brindar posibilidades reales de formación y respuesta a necesidades de maestros y estudiantes, amparadas en lo pedagógico y lo humano, para que así, la educación pueda aportar a la autorrealización de los sujetos, lo cual implica la inclusión de lo afectivo, lo ético, lo estético, lo físico en los programas educativos (Niño Zafra, 2005). En consecuencia, las acciones de maestros y estudiantes en diversos espacios serían más asertivas, ya que los maestros serían ejemplo de lo que enseñan, al ser proveídos de las mismas experiencias que se espera ellos promuevan en sus estudiantes. Precisamente al darse dichas situaciones, tendríamos más profesionales de la docencia dedicados no sólo a "ser dictadores de clase", sino a escuchar, comprender, respetar, tolerar, guiar y crecer junto a otros como seres humanos.

En el desarrollo del proyecto se toma la perspectiva elaborada en su tesis doctoral por Niño Zafra (2001), donde se analizan las posibilidades de concebir los estándares de desarrollo profesional de los profesores, revisando los supuestos conceptuales sobre los que se 
apoyan, superando los problemas de los estándares impuestos desde instancias externas, transformándolos en un colectivo de trabajo para la toma de decisiones sobre el aprendizaje de los estudiantes; así como, la compresión del fortalecimiento de las capacidades de la comunidad educativa.

En esta misma dirección Cabra (2008: 97) plantea el "sentido regulador y orientador que podrían tener lo que denomina como estándares de práctica en tanto principios que pueden activar iniciativas de mejoramiento docente y profesional" con la intención de potenciar el trabajo colectivo y público de la evaluación, así como la importancia de su puesta en práctica, conocimiento y responsabilidad profesional en torno a la elaboración, orientación y reorientación de los currículos.

De cualquier manera, aunque se trate de una reflexión autónoma, se requiere de la elaboración cooperativa, respondiendo al potencial que tiene el docente como investigador crítico de la realidad y responsable de su rol profesional de la educación. Estas formulaciones teóricas se han tomado en cuenta como marco de referencia para la propuesta metodológica sobre el sentido y significado dados a las categorías estándares, currículo y evaluación en la indagación a pares académicos nacionales e internacionales, así como en la participación de profesores de colegios distritales con sus conocimientos y experiencias.

\section{Perspectiva metodológica}

Para abordar la reflexión se han agrupado las respuestas a partir del pensar reflexivo, razonable y práctico, orientado al sentido y a la búsqueda constante de significado, para fortalecer los valores y ampliar la comprensión entre sujetos con una actitud hacia el diálogo y la democracia. En este contexto, la evaluación tiene como propósito comprender fenómenos desde los sentidos y significados de un contexto situado, que posibilita el desarrollo como seres humanos a través de la acción y el lenguaje.

En la perspectiva metodológica, el presente trabajo optó por la visión hermenéutica (Martínez 2010) entendida como la comprensión e interpretación del significado dado a los estándares y sus relaciones con el currículo y la evaluación. Se focalizó en la investigación evaluativa de carácter cualitativo, cuya finalidad es comprender el sentido y significado de las interacciones sociales en contextos específicos y entender la multiplicidad de sentidos, redes, relaciones, versiones e intencionalidades de los Estándares con el Currículo y la Evaluación.

Para la indagación teórico-práctica, del proyecto se elaboró una entrevista semiestructurada con los propósitos de identificar y caracterizar los sustentos conceptuales de orden político, administrativo y pedagógico de los estándares, así como identificar y caracterizar las implicaciones y consecuencias que las políticas de estandarización significan para el desarrollo del currículo y la evaluación en la Educación Básica y Media. Esta entrevista contó con diez preguntas que fueron formuladas a seis expertos nacionales e internacionales (Miguel Ángel Santos Guerra, Doctor en Ciencias de la Educación, Catedrático de Didáctica y Organización Escolar en la U. de Málaga, España; Enrique Javier Díez, Doctor en Ciencias de la Educación, Profesor de la U. de León, España; Tiburcio Moreno, Doctor en Pedagogía, Profesor de la U. Autónoma del Estado de Hidalgo, México; Juan Carlos Orozco, Rector de la U. Pedagógica Nacional, Colombia; Guillermo Bustamante, Doctor en Educación, Docente de la U. Pedagógica Nacional, Colombia; José Guillermo Ortiz, Doctor en Historia, Profesor U. Pedagógica Nacional, Colombia.) 
Esta orientación investigativa concede especial importancia al conocimiento y estudio de las opiniones que tienen los participantes, en este caso, los académicos, sobre los temas objeto de estudio y la inclusión de sus respuestas en los Talleres, para la reconstrucción teórica de los mismos.

Asimismo, se ha trabajado el modelo de Eisner que, según Pérez Gómez (1985: 439), consiste en una crítica educativa que toma forma en un documento escrito cuya finalidad es valorar la práctica educativa y sus efectos. Las etapas seguidas en el análisis fueron: la Descripción, la Interpretación y la Valoración, con el propósito de integrar una evaluación crítica, que pueda ser recogida, sistematizada y analizada. Así, fueron descritas e interpretadas las concepciones y prácticas de los docentes sobre los estándares para relacionarlas con el currículo y la evaluación, para develar su sentido y significado en el contexto institucional.

Esta visión cualitativa del conocimiento se dinamiza a través de las siguientes actividades:

1. Desarrollo de talleres con el propósito de orientar el trabajo colegiado que incluye la responsabilidad individual y grupal, que propicie la recolección y retroalimentación de la información en busca de la construcción social del conocimiento.

2. Identificación de opiniones, conocimientos y experiencias de los profesores y de los significados que dan a las categorías de estudio, con el fin de reconstruir sus sentidos.

3. Interpretación de aquella información recogida en los contextos donde funcionan las instituciones educativas con el fin de dar sentido a las expresiones que van más allá de la acción manifiesta.

4. Dar respuesta a las dinámicas particulares de los colegios, privilegiando las situaciones de cada uno de ellos, generando conocimiento idiosincrático y eludiendo la tendencia a generalizaciones mediante fórmulas.

5. Participación de diferentes fuentes: expertos nacionales e internacionales, profesores e investigadores, quienes con sus juicios y argumentos permitieron elaborar una opinión calificada sobre la temática objeto de la investigación.

Esta investigación, por tanto, estudió, analizó y relacionó, a partir del estado del arte, las concepciones de la categoría estándares y su relación con el currículo y la evaluación. Como investigación cualitativa, recolectó datos de forma rigurosa y sistemática a través de talleres, entrevistas formales, semi-estructuradas y encuestas, de manera que se recogiera la voz viva de los participantes de la acción educativa. Finalmente, para el análisis de los datos y la triangulación con la información proveniente de las diferentes fuentes (profesores, directivos, estudiantes, académicos e investigadores), se apoyó en el análisis de contenido y sus fases de categorización, codificación e interpretación, según lo propuesto por Bardin (1999).

\section{Análisis de la información}

En el proceso metodológico del proyecto se asumió la perspectiva de investigación evaluativa con el propósito de investigar el significado dado a la política del Ministerio de Educación Nacional de 2003 relacionado con la formulación de Estándares de Competencias en las Instituciones Educativas Distritales, y buscar, igualmente, el sentido que en los Colegios Heladia Mejía y República de China, los profesores participantes en el estudio han otorgado a tales políticas en la práctica cotidiana. 
Esta visión de investigación evaluativa ha permitido el análisis y una reflexión crítica sobre el sentido que los docentes han expresado de los estándares, así como las implicaciones que dicha comprensión conlleva en los procesos de formación de los estudiantes. Para el análisis de los dos talleres desarrollados con los profesores en los colegios se optó por las etapas formuladas por Eisner (1998): Descripción, Interpretación, Valoración y Tematización.

En cuanto al primer taller titulado ¿Qué son los estándares?, la reflexión se inició con el análisis de las respuestas de los profesores a las preguntas ¿En su Institución Educativa se han tenido, o no, en cuenta los Estándares de Competencia ¿Qué son los estándares y cuáles las razones para que no se hayan utilizado? En esta primera etapa, Descripción, en los Colegios Heladia Mejía y República de China, las respuestas de los profesores son una explicación a la manera en que comprenden las políticas de Estándares del M.E.N. La mayoría de los formatos diligenciados por los 30 docentes (24 formatos) del Heladia Mejía y 80 docentes ( 51 formatos) del República de China, asumieron los estándares en el trabajo de las áreas y, de manera individual, como una reglamentación Institucional emanada del MEN. Sin embargo, sólo son referenciados en el momento de la planeación institucional, pues a lo largo del año no hacen parte importante del P.E.I, razón para que la programación sobre esta categoría quede en los documentos formales de cada aula de clase y de la Institución. Es decir, se perciben sólo como un requisito oficial de comienzo de año.

Asimismo, un número mucho menor de docentes señaló las limitaciones de los estándares en el problema de la homogenización de los procesos curriculares y las limitaciones para dar respuestas a la gran diversidad de necesidades de los estudiantes. Un profesor, incluso, consideró que el docente tiene un papel y una responsabilidad en la adaptación de los estándares de acuerdo con las necesidades regionales y locales de los estudiantes.

La Interpretación a estas respuestas permite entender, a la luz de los referentes teóricos y prácticos, qué está sucediendo con la política sobre estándares. En el caso de los profesores de la Institución Heladia Mejía, no hay un consenso en sus opiniones. En contraste, en las respuestas de los académicos y los teóricos consultados en este proyecto, los estándares se plantean como una política educativa en evaluación que tiene como intencionalidad el control curricular, la medición de logros en pruebas nacionales de colegios y escuelas bajo el lema de alcanzar la calidad de la educación. El pensamiento de los profesores puede derivarse de lo sostenido desde otra perspectiva, por Organismos Internacionales, como la Comisión Internacional sobre Educación, Equidad, y Competitividad Económica (PREAL) en las políticas educativas para América Latina en 1989, donde se establece la urgencia de los estándares para medir a los sistemas educativos, para lo cual se requiere que los estudiantes participen en pruebas nacionales e internacionales. En este marco de referencia, en Colombia, la normatividad del Decreto 1290 de 2009 sobre evaluación de los estudiantes parece estar en consonancia con estas directivas, por lo que en su Artículo Primero, se declara el mandato de adoptar tales directrices, restando importancia al propósito fundamental de dicha norma: la generación de autonomía institucional y nacional en el diseño de los Sistemas Institucionales de Evaluación (SIE) de las Instituciones Educativas.

En el caso de la Institución República de China, los estándares se consideran válidos como medición de resultados. Las opiniones de los participantes en el taller se organizan alrededor de cuatro tendencias: 1. Las políticas educativas de los estándares se asocian a los procesos de enseñanza-aprendizaje, por lo que se asumen como parte del currículo y una tarea contractual propia de la escuela - en palabras de Barrantes y otros (2002: 38) "el trabajo docente y lo que acontece en las prácticas pedagógicas, se reduce a un asunto cognitivo que puede ser determinado mediante pruebas estandarizadas" - . 2. Los estándares sirven como pautas para adelantar el proceso de enseñanza y de preparación 
para las pruebas censales de medición. 3. La poca importancia que tienen para los procesos de aprendizaje significativos de los estudiantes. 4. El estándar como una guía externa a la actividad propia de los docentes que debilita la práctica y la transformación pedagógica de las instituciones educativas.

En cuanto a la conceptualización de un estándar, en los dos colegios se coincide en su definición como los mínimos que debe tener un plan de estudios, siendo asumidos por los profesores como los aprendizajes básicos que se plantean por ciclos y grados. Esto coincide con lo presentado por Estrada Álvarez (2003: 52) al afirmar que "la estandarización curricular permite la definición de unos mínimos de calidad, para que las instituciones educativas cuenten con un 'referente común' que asegure a todos los colombianos el dominio de conceptos y competencias básicas para alcanzar desempeños satisfactorios en su actividad laboral, vivir en sociedad y participar en ella en igualdad de condiciones". En este mismo sentido, la PREAL sugiere "que los gobiernos deben establecer estándares educacionales claros, introducir pruebas a nivel nacional y utilizar los resultados para corregir los programas y reasignar los recursos" (PREAL, 2002: 38).

Las respuestas y análisis de autores como Martínez, Noguera y Castro (2003) y del Grupo Evaluándo_nos (2006) conducen a señalar a los estándares como parte de un dispositivo de control y rendición de cuentas, con miras a influir en los resultados de los logros y desempeños de los estudiantes en las pruebas estandarizadas. Las limitaciones presentes en la conceptualización acerca del significado y finalidad de los estándares pudieran explicarse, de un lado, a la falta de estrategias del MEN para hacer que los profesores se apropien y den sentido a estas políticas y, de otro lado, a la exclusión de los profesores en el diseño, la planeación, el conocimiento y puesta en acción de las mismas.

La Valoración para Monedero (1998) es el ejercicio de comprensión o reconocimiento de las características que tienen los procesos de enseñanza, que para el equipo investigador tiene que ver con los sentidos dados al concepto de estándar: la forma como ha sido formulado por el MEN y abordado por los docentes en los colegios y su caracterización. Esta apreciación se apoya en varias de las investigaciones del Grupo y planteamientos de la pedagogía critica así:

- La puesta en marcha de unas políticas educativas que ven en la evaluación una manera directa de controlar los contextos educativos según las necesidades del mercado, a través de una efectiva aplicación de los estándares de competencia.

- Los lineamientos emanados del MEN se sustentan en la visión técnico-instrumental, descritos como normas que son ajenas a la realidad del contexto de los maestros. Esto influye negativamente en la práctica del estudio y discusión colectiva sobre lo que los estudiantes deben saber, sobre los sentidos dados a los estándares y la participación de los docentes en dicha discusión. Para los estudiantes, los estándares constituyen un referente de medición de los contenidos desde asignaturas concebidas aisladamente y dentro de una jerarquización determinada por las pruebas estandarizadas; es decir, unos contenidos parcializados y distantes de las problemáticas de las comunidades educativas, que enfatizan la competencia externa y la competitividad entre los estudiantes.

- El docente en su rol profesional ve amenazada su autonomía al considerarse su trabajo como un funcionario aplicador de normas y disposiciones ajenas a su labor cotidiana, como un operario traductor de contenidos formulados en normatividades oficiales, manuales de procedimiento y guías instrumentales de casas editoriales. 
- Una reflexión sobre el fortalecimiento de la profesión docente señala la necesidad de la organización colectiva de los profesores en asociaciones o sindicatos para el estudio crítico sobre problemáticas propias del trabajo pedagógico, entre ellas, los estándares de competencia que faciliten el reconocimiento del rol del profesor como intelectual reflexivo, crítico, propositivo con proyectos alternativos que fomenten la creatividad y generen transformaciones pedagógicas.

- Se requiere la visión de los estándares desde la perspectiva que tenga en cuenta la diversidad de contextos socioculturales de los estudiantes, de su formación como personas autónomas, críticas y creativas con sentido crítico para asumir el mundo globalizado, superando los intereses neoliberales (del hombre para la producción) y neoconservadores (del hombre sometido a las exigencias de las pruebas, exámenes y estándares) al concebir al estudiante y al profesor como sujetos activos que hacen parte de comunidades de aprendizajes significativos que propicien el acceso a bienes simbólicos-culturales que posibiliten las transformaciones sociales y educativas.

En cuanto a las posibilidades que ha generado la implementación de esta política de estandarización, se puede deducir de las opiniones de los profesores que los estándares permiten la movilidad de los estudiantes entre colegios y ciudades. Asimismo, facilita la concreción del plan de estudios, así como la unificación de criterios, temáticas y conceptos para girar en torno a una base conceptual homogénea. De esta manera, permite cuestionar su propia labor al plantear interrogantes sobre el qué, el cómo y lo que es significativo en el proceso de enseñanza-aprendizaje.

En particular para la Institución República de China, las posibilidades de la política de estándares se centran en que los docentes participantes podrían trascender o complementar el sentido que tiene la puesta en marcha de los estándares. De acuerdo con sus comentarios, en primer lugar, es necesario superar el lenguaje técnico-instrumentalista con que están diseñados, planteando además la necesidad de formular estándares para todas las áreas sin discriminación. En segundo lugar, se pueden deducir limitaciones en la aplicación de estas políticas. Esto se ilustra con el interés en la homogenización de las escuelas al desconocer las diferencias en los contextos sociales e individualidades de los estudiantes; la prioridad que se le brinda a las áreas de matemáticas, ciencias y lenguaje, dejando en otro lugar otras dimensiones valiosas para la formación integral del ser humano; la inexistencia en la claridad sobre el significado que tienen los estándares en la labor docente, posiblemente debido a una falta de mayor apropiación y comprensión de los mismos. Esto confirma que los estándares tienden a la homogenización de los aprendizajes, lo que sin duda hace que la evaluación se convierta en un estándar de segregación y clasificación de estudiantes.

En el segundo taller titulado Hacia la conceptualización del sentido dado a los estándares: ¿Qué proponen los profesores y directivos de Instituciones Educativas?, los análisis se dieron en torno a las respuestas de los pares académicos nacionales e internacionales sobre la pregunta ¿Qué concepto le merece la política del Ministerio de Educación de su país, acerca de la formulación de los estándares en educación y, en particular, en las áreas de matemáticas, lenguaje, ciencias naturales, sociales y ciudadanas?", La descripción de las respuestas señala problemas como:

- Homogenización: "medir a todos los estudiantes sin tener en cuenta la diversidad", "se enseña y se evalúa a toda la población de igual manera sin tener en cuenta la diversidad"

- Medición: "se enseña y se evalúa a todos por igual...", "se miden resultados...", "la evaluación estandariza el conocimiento..." 
- Descontextualización: "no tiene en cuenta la cultura ni el momento histórico”, “... no tiene en cuenta las verdaderas necesidades del contexto"

- Administración: "falta compromiso de parte de los estamentos encargados de la planeación y ejecución de los currículos...",

- Desprofesionalización: "no permite la autonomía del docente”, "anula la creatividad del estudiantes y del docente"

La Interpretación de este segundo taller favorece el análisis sobre los problemas de los estándares. Tales reflexiones de los docentes se pueden sintetizar de la manera en que se presentan a continuación:

- Imposición de la normatividad: estas políticas dificultan las transformaciones pedagógicas en las instituciones al llegar como imposición externa sin posibilidad de análisis en su implementación. Sin embargo, un grupo de docentes ve posibilidades para estas transformaciones, pues los contenidos de los estándares se organizan en grados y temáticas en cada año escolar.

- Control de resultados: a través de la evaluación se controla el proceso de formación de los estudiantes mediante la aplicación de los estándares en un ejercicio de "utilización del sistema educativo como forma de adoctrinamiento", tal y como señaló el profesor Enrique Díez en su entrevista. Mientras que la educación que se fundamenta en los estándares y los exámenes (test) según Giroux, citado por Chomsky (2007) “desatiende el aprendizaje que ha de guiar el yo con la vida pública y de la responsabilidad social con las peticiones más generales de la ciudadanía" (Chomsky, 2007: 10).

Como Valoración, se podría concluir que la puesta en marcha de la política de estandarización en América Latina, particularmente en la primera década del siglo XXI, ha sido el resultado de unas políticas educativas pensadas desde la perspectiva económica y política y, mediada por el capitalismo que mediante la globalización de la ideología neoliberal viene desplegando todo su poder, con el fin de imponer un dispositivo social capaz de hacer control y vigilancia a todos los sistemas educativos latinoamericanos. Todo esto se hace a través del currículo único, la evaluación como rendición de cuentas de las instituciones y de los individuos; desde la misma "despedagogización" y desprofesionalización de la profesión docente y la creciente política de desprestigio de la educación pública. Son estas las banderas que han cambiado la brújula de la formación de calidad en la educación.

\section{Tematización y conclusiones}

Una vez sistematizada y analizada la información obtenida en el desarrollo de los talleres con los profesores y de las entrevistas realizadas con seis reconocidos académicos nacionales e internacionales, expertos en el tema de los estándares en educación, se han construido unas conclusiones surgidas de las múltiples relaciones generadas desde los códigos y categorías definidas en el marco teórico, así como aquellas emergentes en el proceso metodológico en un panorama global de sentido y significado construido en la acción investigativa. Esta diversidad y amplitud de visiones, reconocen el valor de la particularidad de cada elemento analizado y brindan mejores comprensiones sobre los problemas investigados. Para el Grupo, la reflexión desde la intencionalidad que guardan en sí mismas las políticas educativas en evaluación, favorecen el conocimiento sobre las vivencias y experiencias de los sujetos e instituciones que participaron en la investigación. 
Estas intencionalidades se derivan de las políticas educativas en evaluación provenientes de la economía política neoconservadora y neoliberal propias de la globalización, en búsqueda del control del saber de los sistemas educativos tanto en el contexto mundial como en los contextos nacionales. Reformas educativas que son construidas e implementadas en el marco de una economía de mercado definido por Organismos Internacionales como el Banco Mundial, Banco Interamericano de Desarrollo y, en los últimos 20 años de la Organización para la Cooperación y el Desarrollo Económico - OCDE, influyen en los países y concentran su interés en medirlos y compararlos entre sí.

Colombia no ha sido exenta de este proceso mundial, por lo que ha adecuado el Sistema Educativo Nacional a las demandas sociales, políticas y económicas que implementan el modelo de capital y desarrollo humano para la formación de niños, jóvenes y adultos. Este modelo se sustenta en los programas de los Gobiernos y los planes sectoriales de Educación, entre otros, lo que termina por ejercer el control total del currículo, las prácticas pedagógicas, la evaluación y los sujetos que se involucran en la institución educativa, desconociendo las particularidades de su contexto interno y externo. En este marco de políticas educativas desde el modelo neoliberal se afianzan valores político-administrativos de eficiencia, eficacia y competitividad, que se utilizan en las directrices ministeriales para implantar las "competencias y los estándares en educación" en el currículo y la evaluación en todos sus niveles. Esto promueve la instauración del modelo técnico de educación que contiene, a su vez, consecuencias inmediatas instrumentales y operativas de todos los sujetos educativos (estudiantes y docentes).

En Colombia, a partir del 2002, el lugar predominante de los estándares define un modelo de educación impuesto que desconoce las diferencias entre los estudiantes, así como su contexto y las realidades locales, regionales, nacionales e internacionales. Este desconocimiento del contexto evidencia la homogenización y el control a través de las evaluaciones nacionales e internacionales. En los colegios, una de las características más significativas encontradas es la de considerar los estándares como mínimos de contenidos del plan de estudios que no hacen parte de la programación curricular a lo largo del año. En segundo lugar, la política educativa de estándares, en coherencia con las normas de evaluación educativa -Artículo $1^{\circ}$ del Decreto 1290 del 2009-, busca medir los estándares educativos a través de un dispositivo de control y de rendición de cuentas.

En tercer lugar, la aplicación de los estándares promueve la educación fragmentada en disciplinas, desconociendo el valor de la integración de los conocimientos y segregando las áreas que no son catalogadas como fundamentales. Esto se evidencia en lo expuesto por los profesores, quienes señalan que la política de estándares "no permite que los colegios ni los docentes sean innovadores ni autónomos, porque deben apuntar a una evaluación donde se miden solamente conocimientos y no se tiene en cuenta el desarrollo individual de los estudiantes y anula la creatividad del estudiante y del docente, según comentario del grupo de docentes participantes en el primer y segundo taller desarrollados en las Instituciones Educativas.. Esta situación coincide con descripciones de Santos Guerra (2010), quien anota: "he visto escuelas que han dejado programas de coeducación, de educación para la paz, de ecología, con miras a conseguir buenos resultados en pruebas estandarizadas".

En cuarto lugar, la profesión docente es reducida a labores técnicas a partir de la aplicación de las normas sobre estándares según las áreas del conocimiento, lo que hace del currículo un elemento pre-establecido, sometiendo al profesor a la competitividad entre instituciones y docentes con el fin de mejorar los resultados en las pruebas externas y que conduce al abandono progresivo de la formación integral de los estudiantes, a cambio de prestigio social obtenido por la publicación de los rankings anuales emitidos por el Instituto 
Colombiano para la Evaluación de la Educación - ICFES. En este marco, el docente es desconocido como profesional e investigador de la educación y la pedagogía, lo cual promueve la desprofesionalización y despedagogización de su labor.

En relación directa con la problemática objeto de estudio, los efectos adversos de los estándares, las competencias y los lineamientos curriculares como elementos del dispositivo evaluador del Estado, comportan consecuencias en la autonomía escolar, la profesionalidad del docente, la pedagogía y la formación integral; razones que han llevado a los maestros participantes de este ejercicio investigativo a la re-significación del conocimiento académico sobre su acción evaluativa y su práctica docente. Esto genera una reflexión sobre las limitaciones y problemas de los estándares, por lo cual resulta conveniente que los estándares sean comprendidos como orientaciones y no como imposición, y desde allí realizar un trabajo pedagógico que reconozca la diversidad sociocultural y la no igualdad de oportunidades con relación a los procesos educativos de los sujetos y a la calidad de la educación que se oferta.

En este sentido, urge una apropiación sobre el sentido dinámico de la evaluación como parte de la formación integral que rescata la multidimensionalidad del ser humano, considerándola como elemento fundamental del sistema educativo que permite superar la medición, el control y la homogenización. Estas necesidades desde las propuestas de los docentes que integran las instituciones educativas, buscan ampliar y desarrollar prácticas alternativas de formación democrática a través de ejercicios como la autoevaluación y coevaluación de estudiantes, así como la construcción participativa del currículo.

\section{Bibliografía}

Álvarez, J.M \& López, J. (1999) La Evaluación del Profesorado y Equipos Docentes. Madrid: Síntesis educación.

Bardín, L. (1997) Análisis de contenido. Madrid: Akal.

Cabra, F. (2008) La calidad de la evaluación de los estudiantes: un análisis desde estándares profesionales. Revista Internacional de Investigación en Educación, Colombia: Pontificia Universidad Javerianav.1 fasc. p.95 - 112

Darling-Hammond, L. (1995). Linda Darling-Hammond, Jacqueline Ancess, and Beverly Falk New York: Teachers College Press. Localización Uned Biblioteca Central 1371.26 DAR. Monografía.-

Eisner, E. (1998) El ojo ilustrado. Barcelona: Paidos Educador

Elliot, J. (1986). Autoevaluación, desarrollo profesional y responsabilidad. EN: Galton M. y Moon B.: Cambiar la escuela, cambiar el curriculum. Barcelona: Martínez Roca. 237259.

Gimeno-Sacristán, J. \& Pérez-Gómez, A. (1986) La enseñanza: su teoría y su práctica. Madrid: Akal Universitaria.

Kemmis, S. \& McTaggart, R. (1992) Cómo planificar la Investigación - Acción. Barcelona: Laertes.

López, M. (1995) Lecturas de metodología histórico educativa. Valencia: Martín impresores.

McKenna, B. \& all. (1998) Guía Profesional para la Mejora de los Sistemas de Evaluación del Profesorado -Recursos e Instrumentos Pedagógicos. España: Mensajero.

Monedero, J.J. (1998) Bases teóricas de la evaluación educativa. Málaga: Aljibe 
Nieto, J. (1982). Valoración de la eficacia docente: problemas y técnicas. Serie: Práctica Educativa 21. Madrid: Escuela española. Localización UNED Biblioteca Central1 371.13NIE. Monografía.-

Niño, L.S. (2001). Tendencias de Evaluación de Docentes. Revista Opciones Pedagógicas No. 24, Universidad Distrital, Bogotá.

Niño Zafra, Libia Stella (2002). Dimensiones de Evaluación de la calidad de la Educación. Revista Opciones Pedagógicas No.25 Universidad Distrital, Bogotá

Peterson, K.D. (2000) Teacher evaluation. California: CorwinPress.

Santos-Guerra, M.A. (2003) Trampas en Educación. El discurso de la calidad. España: La Muralla

Stenhouse, L. (1998). La investigación como base de la enseñanza. Madrid: Morata.

Villar, L.M. (1987). Evaluación diagnostica de los procesos mentales de los profesores. Sevilla. Servicio de Publicaciones de la Universidad. Localización CIDE. Ejemplares: BM, 371.1 VIL R.13238 - 040867. Número de control: 38000107794

Yaniz, C. (1998) "Un Sistema de Autoevaluación y Mejora para el Profesorado de Educación Primaria". Bilbao, España: Mensajero. 


\section{Autores}

Libia Stella Niño Zafra

Doctora en Pedagogía de la Universidad de Valencia, España. Magister en Psicología Educativa de la Universidad de Wisconsin, EEUU. Coordinadora Grupo de Investigación Evaluándonos desde el año 2002 hasta el primer semestre del año 2013, y docente de la Maestría en Educación de la Universidad Pedagógica Nacional. Ha realizado estudios de investigación en políticas educativas, evaluación educativa, evaluación de docentes, formación de docentes, las relaciones entre currículo, evaluación y formación, entre otros, todos ellos desde las perspectivas críticas y alternativas del conocimiento. Su producción intelectual se plasma en reconocidas revistas como Opciones Pedagógicas de la Universidad Distrital, Educación y Cultura de FECODE, Pedagogía y Saberes de la Universidad Pedagógica Nacional, y en libros políticas educativas, evaluación y metaevaluación (2007), De la perspectiva instrumental a la perspectiva crítica. Pedagogía, currículo y evaluación (2010) y Currículo y evaluación críticos: pedagogía para la autonomía y la democracia (2013)

\section{Antonio Gama Bermúdez}

Co-investigador del Grupo Evaluándo_nos, Magister en Educación de la Universidad Pedagógica Nacional. Se desempeña como Consejero en procesos académicos de formación en entornos virtuales de aprendizaje y como asesor pedagógico de diversas entidades en temas como la formación profesional de docentes, la evaluación educativa, el desarrollo curricular, investigación social y entornos de educación virtual y a distancia. Ha participado con el Grupo en el desarrollo de sus investigaciones desde el año 2006 hasta la actualidad. Su producción intelectual se registra en publicaciones como De la perspectiva instrumental a la perspectiva crítica. Pedagogía, currículo y evaluación (2010), Las políticas educativas, evaluación y metaevaluación (2007) y Currículo y evaluación críticos: pedagogía para la autonomía y la democracia (2013) 